\title{
Memoria Histórica, Identidad y Trauma Bericht einer ungewöhnlichen Tagung
}

IreNE PRüPER-LeskE

Universidad de Alicante

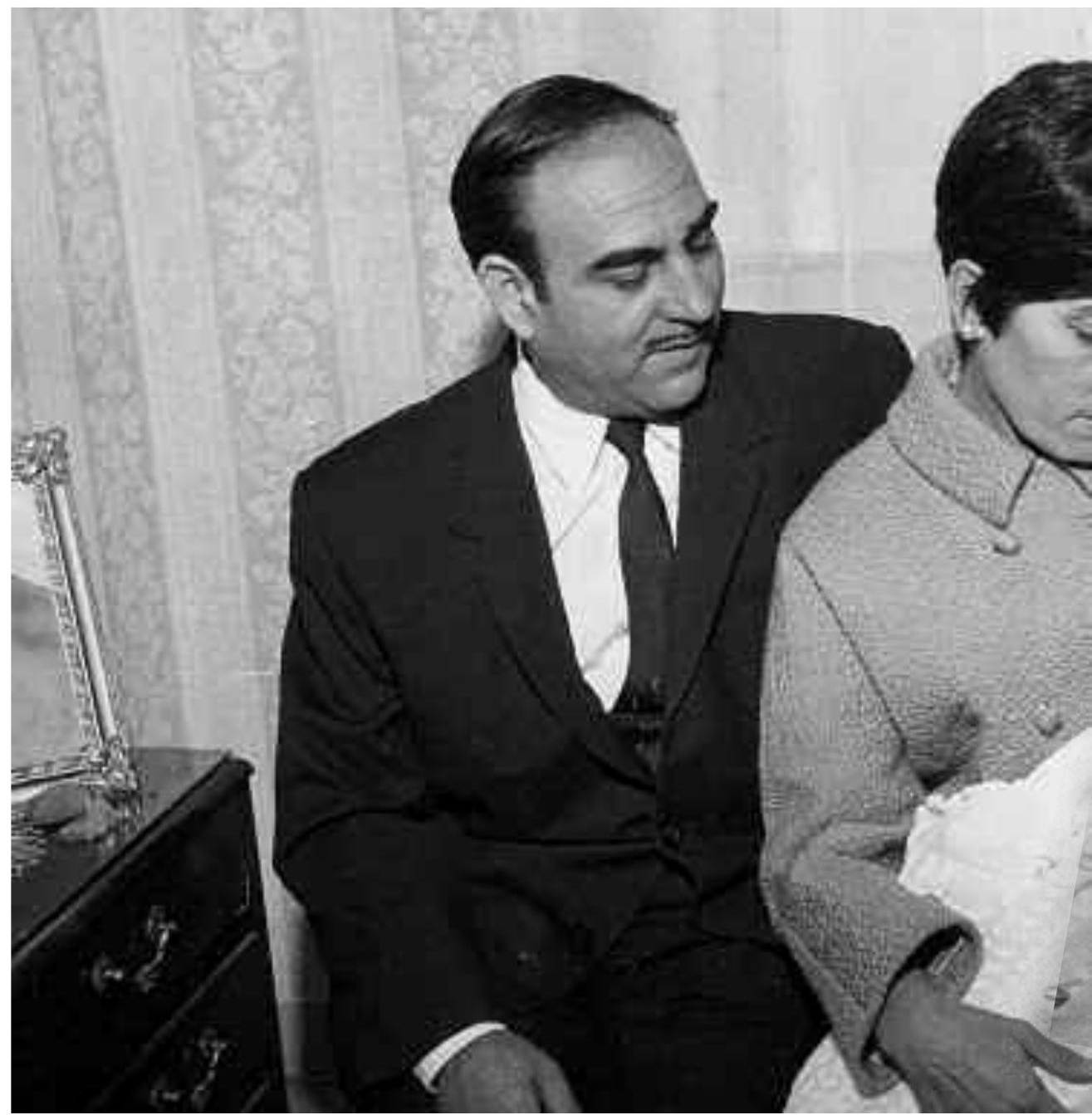

kenaustausch zwischen Kriegskindern des Spanischen Bürgerkrieges, des Zweiten Weltkrieges und den Kindern von Verschwundenen der argentinischen $\mathrm{Mi}-$ litärdiktatur zu ermöglichen. Diese Kinder verschiedener Nationalitäten sind im Zeitraum zwischen den 30iger und den 70iger Jahren des letzten Jahrhunderts geboren. Der gemeinsame Nenner ist bei ihnen der Krieg und/oder der Staatsterrorismus. Wir wollten dabei zwischen dem 14. und 17. September 2011 gemeinsam versuchen, die Bewusstmachung der Geschehnisse und Erlebnisse zu fördern, indem wir uns über kollektives und individuelles Gedächtnis Ge- 


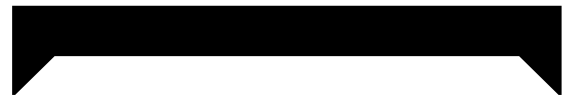

DieTagung befassstesichmit verschiedenen Wissensgebieten im Bereich des kollektiven und individuellen Gedächtnisses, der Verteidigung der Menschenrechte, der Identität, dem Trauma besonders von Kindernund den Auswirkungendieser Thematik auf die Kunst, insbesondere Film, Malerei und Literatur.

diese Weise sollte diesen Generationen Rechnung getragen werden.

Zielgruppen waren also zunächst einmal Betroffene selbst, dann Wissenschaftler und Experten, und weiterhin Menschen, die sich mit Opfern der angesprochenen historischen Ereignisse beruflich befassen (wollen), wie z.B. Ärzte, Psychologen, Rechtsanwälte, Sozialhelfer etc., Krankenschwestern besonders bei der Hilfe von alten Menschen oder Studenten dieser Disziplinen.

Unser Ziel war die Sensibilisierung für Leiderfahrung im Bereich von traumatischen Erlebnissen in der frühesten Jugend, die in vielen Fällen an eine Identitätssuche gekoppelt war.

Die Tagung befasste sich somit mit verschiedenen Wissensgebieten im Bereich des kollektiven und individuellen Gedächtnisses, der Verteidigung der Menschenrechte, der Identität, dem Trauma besonders von Kindern und den Auswirkungen dieser Thematik auf die Kunst, insbesondere Film, Malerei und Literatur. Diese Inhalte wurden von interna- tional anerkannten Experten, aber auch Opfern, erläutert, angesprochen und damit bewusst gemacht und teilweise heftig diskutiert.

Die Fundación Frax in El Albir erwies sich als der geeignete Ort für das Seminar. Die meisten Teilnehmer verschiedenster Herkunft und Nationalität kamen aus dem Umkreis, es waren zum Teil deutschsprachige Residenten, oftmals auch Mitglieder der evangelischen Kirche, die in diesem Teil der Costa Blanca viel humanitäre Arbeit leistet und einen großen $\mathrm{Zu}$ lauf hat. Wünschenswert wäre ein stärkeres Echo unter den Spaniern gewesen, vor allem Betroffenen des spanischen Bürgerkrieges. Einladungen wurden mit den Argumenten abgewiesen, dass man ja kein Trauma habe und dass dieses Kapitel längst abgeschlossen sei und man es besser nicht mehr anrühren solle. Dagegen war die Enkelgeneration, also spanische Studenten sehr interessiert und aktiv beteiligt und vertreten.

Zwei Simultandolmetscherinnen halfen bei Verständigungsschwierigkeiten zwischen deutsch- und spanischsprachigen Vortragenden und Teilnehmern.

Schwerpunkte dieser Tagung an der Costa Blanca bildeten Vorträge mit anschließenden Kolloquien, Augenzeugenberichte, Workshops, Kulturveranstaltungen und eine Buchausstellung. Die Tagung wurde als Veranstaltung der Universität Alicante angeboten, mit der Möglichkeit, 3 Kreditpunkte zu erhalten.

Als Organisatoren und finanziell unterstützende Institutionen fungierten die Universität Alicante, die Stadtverwaltung Alfaz del Pi und die Alexander-vonHumboldt-Gesellschaft der Provinz Alicante mit dem weitaus größten Beitrag. Weitere wertvolle finanzielle Hilfen wur- 
den vom Generalkonsulat der Bundesrepublik Deutschland in Barcelona, von der Konrad-Adenauer-Stiftung Madrid und der Forschungsgemeinschaft 20. Juli 1944 Berlin geleistet. Weiterhin konnten wir mit der Mitarbeit und selbstlosen Hilfe des Amerikahauses Alicante und der Eberhard-Schlotter-Stiftung der Comunidad Valenciana rechnen. Ganz besonders zu erwähnen sind Helmtrud de Roo von Hagen, Tochter des Widerstandskämpfers Albrecht von Hagen im Dritten Reich und Fernando Sandoval aus Argentinien, Sohn von Verschwundenen der Argentinischen Militärdiktatur, die selbstlos und unter Aufbringen von persönlichen finanziellen Opfern bei der Gestaltung der Tagung sehr aktiv mitwirkten. Aber auch alle anderen Vortragenden haben die Philosophie der Tagung erfasst und außerordentlich engagiert ihre Themen und Standpunkte erklärt und lange Anreisen auf sich genommen. Das Außergewöhnliche war, dass fast alle Vortragenden bei der gesamten Tagung anwesend waren, aus den anderen Wissensgebieten der Kollegen neue Erkenntnisse erwarben, ungeachtet dessen, dass nicht alle Vortragende Wissenschaftler waren. Denn hier ging es nicht, wie sonst auf Kongressen um das eigene wissenschaftliche Profil, sondern vielmehr um die schon erwähnten Ziele und Anliegen unter dem Motto „Kriegskinder für den Frieden“. Nicht um Revanche abzuhalten und Schuldige zu finden. Wir wollten erfahren und darüber diskutieren, wie es 75 Jahre nach dem Beginn des spanischen Bürgerkrieges, 66 Jahre nach dem Ende des 2. Weltkrieges, 28 Jahre nach dem Ende der argentinischen Militärdiktatur mit unserem historischen Gedächtnis steht, was wir inzwischen mit dem furchtbaren Erbe und den von Curt Hondrich so genannten Vererbten Wunden wirklich für den Frieden getan haben und dafür, dass alle Ereignisse, die hier zur Sprache kommen sollten, nicht in Vergessenheit geraten und durch die Erinnerung das mahnende Wort „Nie wieder“ von Dachau erhalten bleibt.

Das vorgesehene Programm wurde mit kleinen Abweichungen durchgeführt. Kennzeichnend ist, dass bei der Eröffnung der Tagung kein einziger Vertreter der unterstützenden Institutionen anwesend war.
Im Zentrum des Eröffnungsvortrages des Staatsanwaltes im Obersten Gerichtshof Carlos Castresana stand seine anklagende Haltung gegenüber den bisherigen Regierungen seit der Einführung der Demokratie nach Francos Tod, von denen keine wirklich etwas für die $M e$ moria Histórica in Spanien getan habe: „España siempre ha dejado pasar el tren“. Eine gegenläufige Meinung hatten die beiden Historiker Julián Chaves und Glicerio Sánchez von den Universitäten Extremadura und Alicante, die von ihren Möglichkeiten an den Universitäten und ihren Forschungen in Archiven über den Bürgerkrieg und der heutigen Bewusstseinsveränderung unter der spanischen Bevölkerung berichteten. Demgegenüber sprach der Soziologe Ernesto Espeche von der Universität Del Cuyo in Mendoza / Argentinien, auch Kind kämpfer vom 20. Juli verurteilt wurden, und den Consejos de Guerra unter der Franco-Herrschaft an. Heftige Diskussionen entstanden insbesondere zwischen den Historikern und den Vertretern der spanischen Organisationen pro Memoria Histórica und selbst familiär Betroffenen, Emilio Silva und José Luis Galán, die den Standpunkt vertraten, dass in Spanien bislang nicht genug getan worden sei, um eine wirkliche Diskussion über die Vergangenheit anzuregen und eine Würdigung der Opfer der FrancoDiktatur zu erlangen. Die Arbeit dieser Organisationen und Vereinigungen in Spanien wurden mit z.T. eigens für die Tagung erstellten Videos unterlegt, in denen Meinungen aus den verschiedensten Gesellschaftsschichten zur Worte kamen. Darunter befanden sich auch Augenzeugenberichte, die besonders die jüngeren

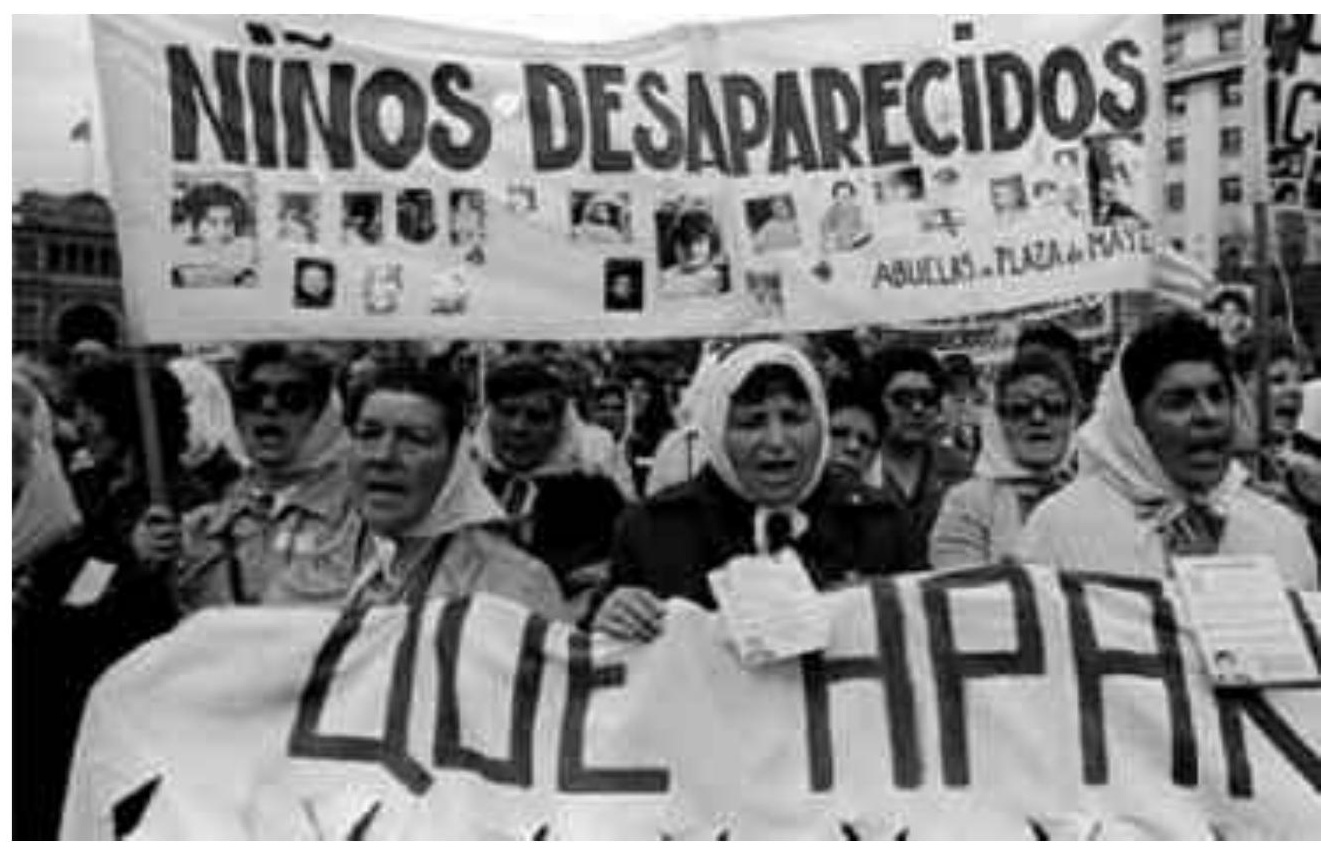

von Verschwundenen, über die Schwierigkeiten beim Entstehen und Aufbau eines kollektiven Gedächtnisses in Argentinien.

Nach dem Vortrag des Historikers Ricardo Martín de la Guardia, Experte für den deutschen Widerstand im Nationalsozialismus und Leiter des Instituto de Estudios Europeos in Valladolid, stellte Glicerio Sánchez einen Vergleich zwischen den Schauprozessen im Volksgerichtshof im Dritten Reich, in dem die Widerstands-
Teilnehmer (Studenten) an dieser Tagung stark beeindruckten.

Damit kommen wir zum zweiten Teil der Tagung, dem der Berichte der Zeitzeugen. Es wurde gelobt, dass die Ablauf der Tagung so angelegt war, dass diese Berichte im Mittelpunkt der Tagung standen, nachdem einführend von historischer und rechtlicher Seite her durch die Experten mit nötigen Informationen und den heutigen Standpunkten auf die Thematik hingeführt worden 
war. Anschließend an die Augenzeugenberichte wurden die psychischen und psychosomatischen Auswirkungen von Psychiatern und Psychologen behandelt, während schließlich am Ende Juristen und involvierte (Menschenrechts-) Organisationen einen Einblick in ihre Arbeit gaben und den Ausblick bildeten.

Es standen jeweils Vertreter der einzelnen Nationen den Teilnehmern zur Verfügung, die ihre Erlebnisse im Bürger$\mathrm{krieg}$, im zweiten Weltkrieg und in der Militärdiktatur in Argentinien vortrugen. Am Anfang stand der Vortrag von Günter Habermann, Mitglied der Forschungsgemeinschaft 20. Juli 1944, dessen Vater sich als Gewerkschaftler und Sozialdemokrat nach der Festnahme durch die Gestapo im Gefängnis das Leben nahm. Nach seinen Ausführungen wurde von Irene Prüfer die Kinderliteratur in

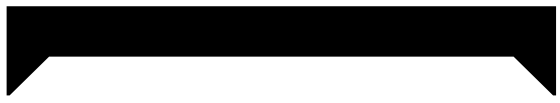

Im Zentrum des Eröffnungsvortrages des Staatsanwaltes im Obersten Gerichtshof Carlos Castresana stand seine anklagende Haltung gegenüber den bisherigen Regierungen seit der Einführung der Demokratienach Francos Tod, von denenkeinewirklichetwasfïrdie MemoriaHistóricainSpanien getan habe.

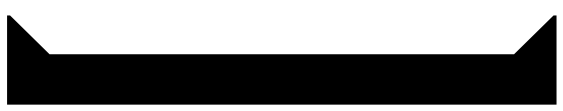

Deutschland und Argentinien unter dem Blickwinkel der Erinnerungskulturen und auf den Einfluss von Kriegskindererlebnissen hin durchleuchtet. Besonders für die jungen Studierenden waren die anschließenden Lebensberichte von Helmtrud de Roo von Hagen (76 Jahre), Tochter von Albrecht von Hagen, der neben Claus Schenk von Stauffenberg am Hitler-Attentat am 20. Juli 44 beteiligt war, von Elisa Villalta Sánchez (81), Nichte von Opfern der Franco-Diktatur und Fer-

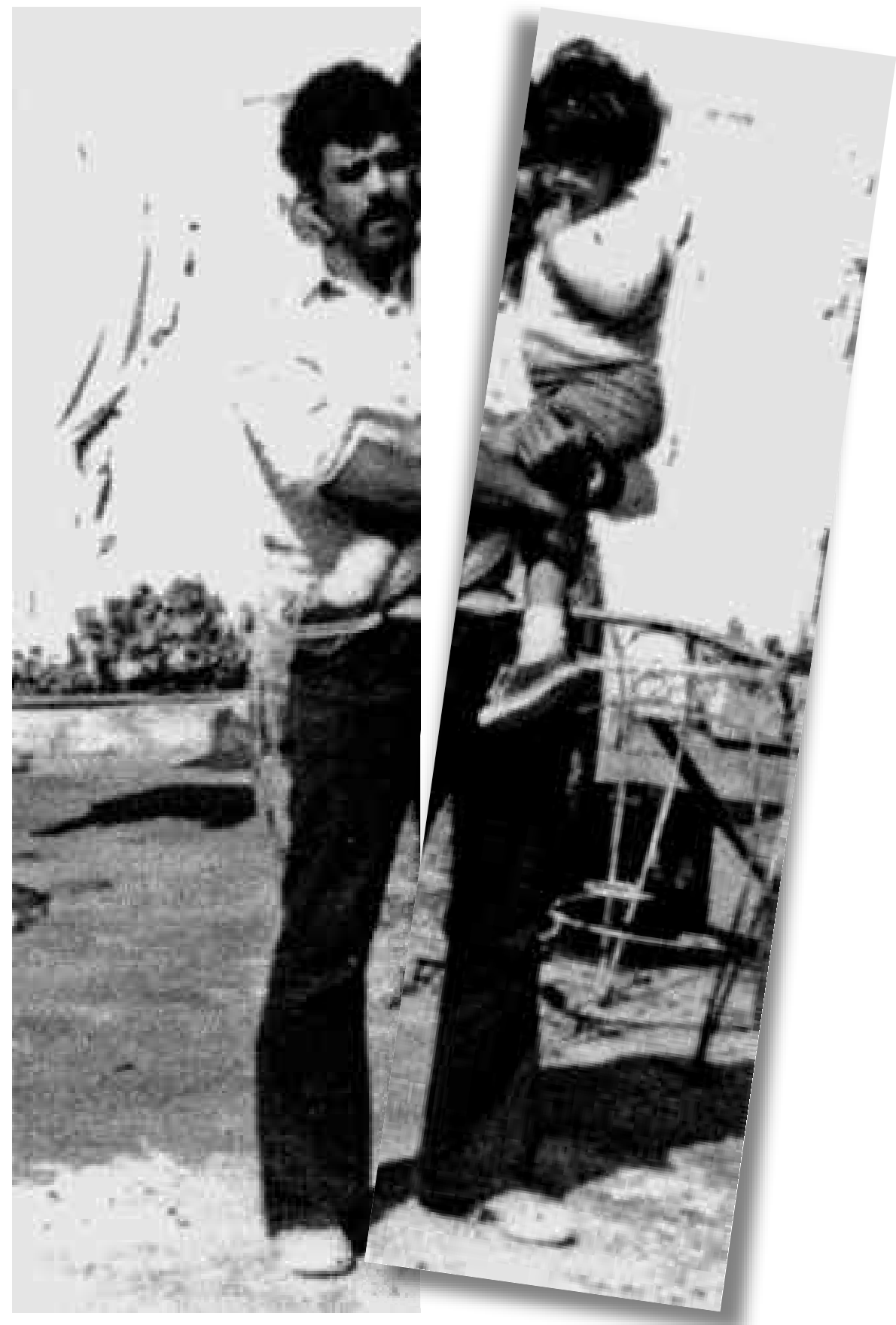

nando Sandoval (41), Sohn und Neffe von Verschleppten der Militärdiktatur in Argentinien (1976-1983) und Halbbruder eines hijo apropiado, sehr beeindruckend. Uns war es nicht leicht gefallen, Zeitzeugen für einen Vortrag zu gewinnen (besonders der Franco-Zeit), denn die persönlichen Schicksale waren ja in allen Ländern lange Zeit Tabu.

In Deutschland sind Seminare dieser Art inzwischen nicht selten. Trotzdem konnten wir den berühmten Psychoanalytiker
Hartmut Radebold mit seinem Thema „Traumata der Kriegskinder“ für einen Vortrag und einen Workshop gewinnen. Die Psychologin Marisa Punta, Professorin der Universität Buenos Aires, gab Einblick in ihre Arbeit als Sachverständige bei Prozessen gegen apropiadores von Kindern, die in der Gefangenschaft und nach der Folterung ihrer Mütter geboren und daraufhin, nach deren Ermorderung, von den Mördern illegal adoptiert wurden.

Die kulturellen Abendveranstaltungen 
mit anschließenden Kolloquien stellten die Themen Kinder und junge Menschen als Leidtragende von Krieg und Staatsterrorismus in den internationalen Blickwinkel von Film, Literatur und Malerei. Es wurde der Film Los girasoles ciegos von José Luis Cuerda mit Untertiteln vorgeführt, Auszüge von Atemschaukel und der spanischen Übersetzung Todo lo que tengo, lo llevo conmigo der Nobelpreisträgerin Herta Müller wurden von zwei jungen Künstlern gelesen und der 91 jährige, in Altea ansässige Maler Eberhard Schlotter und Begründer der Stiftung Eberhard Schlotter de la Comunidad Valenciana führte ein Künstlergespräch über seine Malerei während des Zweiten Weltkriegs in Russland und den Nachkriegsjahren in Deutschland und Spanien.

Trotz des intensiven Programms waren Vorträge und Abendveranstaltungen sehr gut besucht.

Am 17. September, dem letzten Tag, sprachen Vertreter der einzelnen Organisationen über ihre Arbeit: Oscar Strada in Vertretung von FEDEAR (Federación de Asociaciones Argentinas en España y Europa), Emilio Silva als 1. Vorsitzender von ARMH (Asociación para la Recuperación de la Memoria Histórica) und José Luis Galán als Vertreter der Opfer des Franquismus. Am Tag vorher hatte der Psychiater Hartmut Radebold in Vertretung von „Kriegskinder für den Frieden“ über Aufgaben und Ziele dieser Vereinigung gesprochen. Den Schlusspunkt setzte der Staatssekretär für Menschenrechte von Argentinien, Eduardo Duhalde, mit einem Bericht über die gegenwärtigen Maßnahmen seines Ressorts zur Wiedererlangung von Frieden, Recht und Wahrheit.

Unser Ziel, eine Art Zusammenleben während der dreieinhalb Tage von Teilnehmern und Vortragenden zu schaffen und Reflexionen über ein Thema anzuregen, das besonders in Spanien noch weitgehend Tabu ist, nämlich von den Folgen zu sprechen, die Kriegstraumatisierungen auf gesellschaftlich-kulturellem Gebiet in Europa bewirkten, war uns gelungen. Die Kommentare der Studenten, die eine $\mathrm{Zu}$ sammenfassung ihrer Eindrücke und einiger der Vorträge erstellten, waren durchweg positiv. Curt Hondrich, selbst Kriegskind, ehemaliger WDR-Redakteur und Vorsitzender des Fördervereins „Kriegskin- der für den Frieden “, begrüßte unsere Initiative als „einen wichtigen Beitrag zu einer europäischen Erzäblkultur... und besonders, weil mir Aktivitäten zum Thema Kriegskinder in Südeuropa bisher nicht bekannt waren ". Der Student Patricio Vidal beglückwünschte uns „por el curso, el mejor al que he asistido hasta ahora de largo".

Wir danken allen Teilnehmern und Vortragenden für ihr Interesse, ihr Engagement und aktive Mitgestaltung der Tagung und allen oben genannten Institutionen für ihre moralische und finanzielle Unterstützung und Hilfe beim Gelingen derselben.
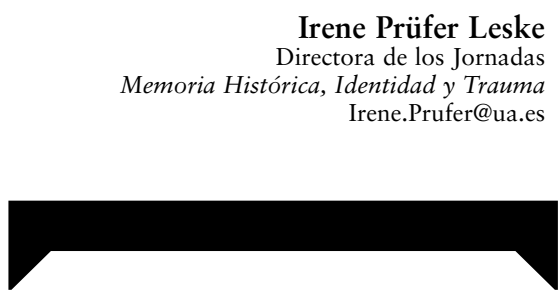

CurtHondrich,selbstKriegskind,ehemaligerWDR-

Redakteur und Vorsitzender des Fördervereins „Kriegskinder für den Frieden“, begrïßte unsere Initiative als „einen wichtigen Beitrag zu einer europäischen Erzählkultur...undbesonders, weil mir Aktivitäten zumThema Kriegskinder in Sïdeuropa bisher nicht bekannt waren“"

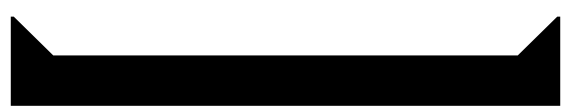

Richtigstellung der Redaktion: Der in der letzten Nummer des magazins (19/2010) veröffentlichte Artikel von Frau Prüfer-Leske, "Un precursor de la pragmática en la enseñanza de lenguas en pleno siglo XIX: el naturista alemán E.A. Rossmässler y sus Cuadros lingüisticos y culturales alemán - español" hätte in seiner deutschsprachigen Version erscheinen sollen. Außerdem musste es natürlich "naturalista" heissen. Fé de erratas: El artículo de la Sra. Prüfer-Leske, publicado en el último magazin (19/2010), «Un precursor de la pragmática en la enseñanza de lenguas en pleno siglo XIX: el naturista alemán E.A. Rossmässler y sus Cuadros lingüísticos y culturales alemán - español» tenía que haberse editado en su versión alemana. Además, naturalmente tenía que ser «naturalista», no «naturista».
Eine Bibliographie

Albrecht, Dagmar (2009): No puedo renegar de mi destino. Albrecht von Hagen y la conspiración contra Hitler de Dagmar Albrecht, traducción de Irene Prüfer, con una introducción de Ricardo Martín de la Guardia "La oposición del ejército alemán al régimen de Hitler y el veinte de julio de 1944". Publicaciones de la Universidad de Alicante.

Bode, Sabine (2005): Die vergessene Generation. Die Kriegskinder brechen ibr Schweigen. München: Piper.

Chmielorz, Rilo (2011): Wie der Krieg lautlos weiter tobt - ein transgenerationales Erbe, http://www.rilochmielorz.de/wie-der-krieg-lautlos-weitertobt-ein-transgenerationales-erbe/

Hondrich, Curt (Hg.): Vererbte Wunden. Traumata des Zweiten Weltkriegs- die Folgen für Familie, Gesellschaft und Kultur. Lengerich: Pabst Science Publishers.

Müller, Herta (2009): Atemschaukel. München: Hanser. Traducción de Rosa Pilar Blanco: Todo lo que tengo, lo llevo conmigo. Madrid: Siruela.

Prüfer Leske, Irene (Hg.) (2010): Die Kinder der Verschwundenen. Augenzengenberichte der argentinischen Militärdiktatur (1976-1983). Stuttgart: Abrazos.

Prüfer Leske, Irene (coord.) (2011): Los hijos de los desaparecidos. Historia colectiva y testimonios de la dictadura militar argentina (1976-1983). Stuttgart: Abrazos.

Radebold, Hartmut (2010): Abwesende Väter und Kriegskindheit. Alte Verletzungen bewältigen. Stuttgart: Cotta.

Sánchez, Glicerio (2010): La República decapitada. El caso de la familia Villalta Gisbert. (Alicante 1939-1942).Barcelona: Les Punxes.

Silva, Emilio (2003): Las fosas de Franco. Crónica de un desagravio. Madrid: Temas de Hoy. 